\title{
Prevalence, clinical presentation and complications of diabetes mellitus in obstetric patients attending a medical college hospital
}

\author{
Prajakta Ganesh Joshi ${ }^{1 *}$, Ganesh Arun Joshi ${ }^{2}$
}

\begin{abstract}
${ }^{1}$ Department of Obstetrics and Gynecology, Chirayu Medical College and Hospital, Bhopal, Madhya Pradesh, India ${ }^{2}$ CRC, Bhopal, Madhya Pradesh
\end{abstract}

Received: 05 July 2017

Accepted: 29 July 2017

\section{*Correspondence:}

Dr. Prajakta Ganesh Joshi,

E-mail: pjosh92@gmail.com

Copyright: (C) the author(s), publisher and licensee Medip Academy. This is an open-access article distributed under the terms of the Creative Commons Attribution Non-Commercial License, which permits unrestricted non-commercial use, distribution, and reproduction in any medium, provided the original work is properly cited.

\begin{abstract}
Background: Diabetes mellitus (DM) may be present in the patient before the conception or it may appear during pregnancy. Obstetric management shall ensure prevention of diabetic embryopathy and early detection and management of diabetic complications in pregnancy.

Methods: A descriptive observational study was undertaken on participants from a Medical College Hospital. The pre-existing medical disorders, blood sugar, routine antenatal investigations, type of delivery, ultrasound findings, complications of delivery, foetal outcome etc. were recorded. The participants were advised diet, exercise and pharmacotherapy. The intranatal and postnatal events were recorded. The results were compared with related literature.

Results: The study had total 89 participants. Five participants (5.6\%) had abnormal blood sugar values. Out of these, 2 participants were having pregestational DM and 3 were having gestational DM. Although all the participants who had abnormal blood sugar levels required caesarean section, two could not be operated. One participant with gestational DM who did not follow management advice delivered a macerated still born baby after shoulder dystocia. Another participant having gestational DM, who complied strictly as per dietary advice and exercise, could be managed well without insulin and delivered a healthy baby. The requirement of insulin increased in pregnancy in patients with pregestational diabetes.

Conclusions: It is essential to ensure compliance on all three pillars of management of diabetes viz. diet, exercise and insulin during pregnancy. Hence health education for diabetes with special emphasis on obstetric care in pregnancy with diabetes should be promoted.
\end{abstract}

Keywords: Gestational diabetes, Obstetric care, Pregnancy in diabetics

\section{INTRODUCTION}

Diabetes mellitus (DM) presents as an important disease condition with $8.5 \%$ prevalence according to global report given by the World Health Organization. In India, it affected $7.8 \%$ population in year 2014 , affecting men more than women and contributed to $2 \%$ of all deaths. ${ }^{1}$ Indian Council of Medical Research has taken it on top priority for research in India identifying rapid increase in incidence of DM from $2 \%$ to $12 \%$ in urban areas over 30 years by turn of the twenty first Century. It estimated that there were 37.8 million diabetics with $4 \%$ rural and $12 \%$ urban prevalence in India in year 2004 with $15 \%$ prevalence of abnormal 3 hour oral glucose tolerance test (OGTT) and hyperglycaemia in urban India. ${ }^{2}$

Diabetes may be present in the patient before the conception in the form of Type 1 or Type $2 \mathrm{DM}$ or it may 
appear during pregnancy as gestational DM. DM can have disastrous consequences for both the foetus and the mother if not treated.

With increasing cases of Type $2 \mathrm{DM}$ and better survival and achievement of obstetric career by Type 1 DM patients; the obstetricians are frequently facing the challenge of preventing and reducing congenital impairments ensuring a healthy baby to add happiness to the family. Both hypo and hyperglycemia is associated with embryopathy in animal studies but in humans only hyperglycemia has been implicated. Incidence of diabetic embryopathy is $3 \%-8 \%$. Patients with DM are reported to have $30 \%-50 \%$ incidence of foetal losses in various studies. ${ }^{3}$ Obstetric management shall ensure prevention of diabetic embryopathy and early detection and management of diabetic complications in pregnancy.

DM complicating pregnancy may give rise to infections, pregnancy induced hypertension, thrombo-embolic episodes and worsening of diabetic nephropathy and retinopathy. The result may be abortion, macrosomia, intrauterine death, complicated labour, and post partum bleeding. Thyroid dysfunction should also be evaluated and monitored in cases with DM. Prevention and management of DM during pregnancy includes screening for DM, glycaemic control, regular ante-natal checkups, appropriate timing of delivery. Incidence of caesarean section in DM complicating the pregnancy is as high as $50 \%$ and the patient should be never allowed to cross expected date of delivery due to the risk of unexplained late fresh still births and the complication of shoulder dystocia. ${ }^{4}$ Watchful screening for diabetes and lifestyle modification in later life is advocated.

Pre-existing diabetes with uncontrolled blood sugar is a major cause of congenital malformations of the newborn. Diabetic embryopathy is associated with anencephaly, spinal dysraphism, congenital heart disease (transposition of great vessels, ventricular septal defect, coarctation of aorta, atrial septal defect), renal agenesis and anal atresia. It may cause hypoglycaemic brain injury and sudden death of the infant. Chances of congenital anomalies are $20-25 \%$ if glycosylated haemoglobin levels in blood are more than $8.5 \% .^{4}$

The management includes strict glycaemic control, taking into consideration, gastro paresis specific to pregnancy and dietary indiscretions because of the pregnant state. Foetal screening and perinatal monitoring is also essential. Institutional delivery with neonatal support is advocated and newborn's blood glucose level should be checked $^{3}$. Baby friendly clinic practices and developmental screening are essential in follow-up to detect and manage developmental disability. Risk factors for GDM include greater maternal age, higher BMI, member of an ethnic group at increased risk for development of type 2 DM (i.e., Hispanic, African, Native American, South or East Asian, or Pacific Islands ancestry), polyhydramnios, past history of GDM, macrosomia in a previous pregnancy, history of unexplained stillbirth, type 2 diabetes mellitus in a first degree relative, polycystic ovary syndrome, and metabolic syndrome. ${ }^{5}$ With the available guidelines, DM can be managed well with diet, exercise and insulin supplementation as the three main pillars of treatment. Patient compliance and cost of medical management of diabetes are the most important factors that impede ideal and possible management of diabetes in gestation. With a curiosity to learn about the diabetes reporting to medical college and results of its management according to available guidelines, we planned to take up this study. Here, we delve into ascertaining hospital prevalence of diabetes in pregnancy, observing the course of pregnancy with DM and sharing the experience in managing the patients.

\section{METHODS}

A descriptive observational study was planned on participants from a Medical College Hospital. Following the ethical guidelines laid out by World Medical Association's Declaration of Helsinki, 89 patients admitted in Labour room of Chirayu Medical College and Hospital, Bhopal was studied during July 2013 to December 2014. The participants included both ANC registered and emergency patients reporting for institutional delivery at the hospital. However, the patients who did not achieve duration of gestation of 28 weeks were excluded.

The type of delivery, foetal outcome, complications of delivery, ultrasound findings, pre-existing medical disorders and blood sugar was recorded. Random blood sugar was done in all participants. In participants having family history, high random blood sugar and polycystic ovarian disease glucose tolerance test was done. The Carpenter and Couston criteria were used to screen gestational diabetes in high risk patients. ${ }^{6}$ In the participants having pregestational diabetes, HbA1c was measured at 6 weeks, transvaginal ultrasound was done at 10 weeks for measurement of nuchal translucency, triple test and anatomical scan at second trimester were done to rule out congenital anomalies.

The participants were advised diet consisting of $50 \%$ calories from complex carbohydrates, $10 \%$ from fats (mainly unsaturated) and 30\% from proteins with fibre rich green leafy vegetables, fruits and legumes. The daily diet was distributed as three major meals and three snacks. Intake of simple sugar, refined flour and potatoes was restricted. Exercise was prescribed with walking for 45 minutes daily at least 5 times a week.

Two of the participants had pregestational diabetes and were on insulin supplementation. One of the three participants having gestational DM also needed insulin. Four hourly blood glucose monitoring was done in all the three participants taking insulin from 32 weeks of gestation. Insulin was given with regular adjustment of 
dose in consultation with medical specialist. The management of labour, intranatal and postnatal events were recorded. The results were compared with related literature.

\section{RESULTS}

The study had total 89 participants whose age ranged from 19 to 37 years with a mean age of 25.66 years. Most of the participants were from urban background, only 14 hailed from the rural areas.

Forty four mothers $(49.5 \%)$ were primigravida. The duration of gestation varied from 28 weeks to 42 weeks with mean of 38 weeks. Ninety two babies were delivered including twins from 3 participants and the baby weight ranged from $1.3 \mathrm{~kg}$ to $3.8 \mathrm{~kg}$ with mean of $2.696 \mathrm{~kg}$. Two babies were still born $(2.2 \%)$.

Table 1: Profile of participants with abnormal blood sugar during pregnancy.

\begin{tabular}{|c|c|c|c|}
\hline Random blood glucose & Glucose tolerance test & HbA1c & Management and course \\
\hline 285 & Not done & 8.4 & $\begin{array}{l}\text { Known diabetic on regular insulin. Delivered } \\
\text { monochorionic diamniotic IUGR twins at } 37 \\
\text { weeks by emergency caesarean section. First of the } \\
\text { twin had friable umbilical cord and thick } \\
\text { meconium stained liquor. Had mild post partum } \\
\text { haemorrhage. }\end{array}$ \\
\hline 222 & Not done & 7.97 & $\begin{array}{l}\text { Diagnosed as Gestational Diabetic, given regular } \\
\text { insulin. Delivered preterm baby } 1.99 \mathrm{~kg} \text { at } 33 \\
\text { weeks by emergency caesarean section for } \\
\text { bicornuate uterus and PROM. Normal blood sugar } \\
\text { after delivery. }\end{array}$ \\
\hline 193 & $70,196,159,100$ & 7.4 & $\begin{array}{l}\text { No treatment could be initiated due to non } \\
\text { compliance. Delivered } 3.8 \mathrm{Kg} \text { macerated still born } \\
\text { baby with shoulder dystocia and cord around neck } \\
\text { at } 38 \text { weeks. Had vaginal lacerations with } \\
\text { haematuria for half hour after delivery. }\end{array}$ \\
\hline 126 & Not done & 6.37 & $\begin{array}{l}\text { Known diabetic on regular insulin with good sugar } \\
\text { control. Delivered IUGR baby with } 2 \mathrm{Kg} \text { birth } \\
\text { weight that needed NICU admission. }\end{array}$ \\
\hline 108 & $98,193,163,138$ & 6.2 & $\begin{array}{l}\text { Strict diet control. Recurrent furuncles in last } \\
\text { trimester of pregnancy. }\end{array}$ \\
\hline
\end{tabular}

Table 2: Comparison of participants with normal blood sugar and abnormal blood sugar.

\begin{tabular}{|c|c|c|}
\hline Variable studied & Non-diabetic (n=84) & Diabetic $(n=5)$ \\
\hline Urban background & $60(83.3 \%)$ & $5(100 \%)$ \\
\hline Age range & 19 to 37 years Mean $=251 / 2$ & 24 to 31 years Mean $=28$ \\
\hline Gravidity (more than one) & $42(50 \%)$ & $3(60 \%)$ \\
\hline Anaemia & $10(11.9 \%)$ & 0 \\
\hline Medical disorder & $17(20.2 \%)$ & $3(60 \%)$ \\
\hline Abnormal USG findings & $16(19 \%)$ & $3(60 \%)$ \\
\hline Intrauterine growth retardation & $10(11.9 \%)$ & $2(40 \%)$ \\
\hline Family history of diabetes & 0 & $3(60 \%)$ \\
\hline Urinary tract infection & $2(2.3 \%)$ & $1(20 \%)$ \\
\hline Medications during pregnancy & $9(10.7 \%)$ & $3(60 \%)$ \\
\hline Pre-eclampsia & $16(19 \%)$ & $2(40 \%)$ \\
\hline Duration of gestation & 28 to 42 weeks Mean $=38$ & 33 to 39 weeks, Mean $=37$ \\
\hline Premature rupture of membranes & $11(13.1 \%)$ & $3(60 \%)$ \\
\hline Caesarean delivery & $65(77.4 \%)$ & $4(80 \%)$ \\
\hline Babies with cord around the neck & $11(13.1 \%)$ & $2(40 \%)$ \\
\hline Meconium stained Liquor & $9(10.7 \%)$ & $2(40 \%)$ \\
\hline Baby weight & 1.3 to $3.8 \mathrm{Kg} ;$ Mean $=2.7$ & 1.9 to $3.8 \mathrm{Kg} ;$ Mean $=2.5$ \\
\hline Still birth & $1(1.2 \%)$ & $1(20 \%)$ \\
\hline Babies admitted to NICU & $9(10.6 \%)$ & $3(75 \%)$ \\
\hline Postpartum haemorrhage & $3(3.6 \%)$ & $3(60 \%)$ \\
\hline
\end{tabular}


Eighty four participants had normal blood sugar values and had no family history or other risk factors for diabetes. Five out of 89 participants $(5.6 \%)$ had abnormal blood sugar values (Table 1). Out of these, 2 participants were having long standing DM and were taking Insulin regularly. Eighteen participants $(20.2 \%)$ had some or the other medical disorder including anaemia. Anaemia was present in 10 participants (11.2\%). Three (3.4\%) participants had urinary tract infection. Twelve participants (13.5\%) were on medications during pregnancy. Ultrasonography findings were normal in 69 participants $(77.5 \%) .12$ participants $(13.5 \%)$ had intrauterine growth retardation of foetus. Sixty-eight participants $(76.4 \%)$ delivered by caesarean section. Fourteen participants (15.73\%) had premature rupture of membranes. Liquor was meconium stained in 11 participants $(12.4 \%)$. Thirteen participants $(14.6 \%)$ gave birth to babies with cord round the neck. Thirteen babies (14.5\%) required admission to NICU. Six participants $(6.7 \%)$ had postpartum haemorrhage. Comparative details of the participants having abnormal blood glucose levels during pregnancy against those having normal blood glucose levels are given (Table 2).

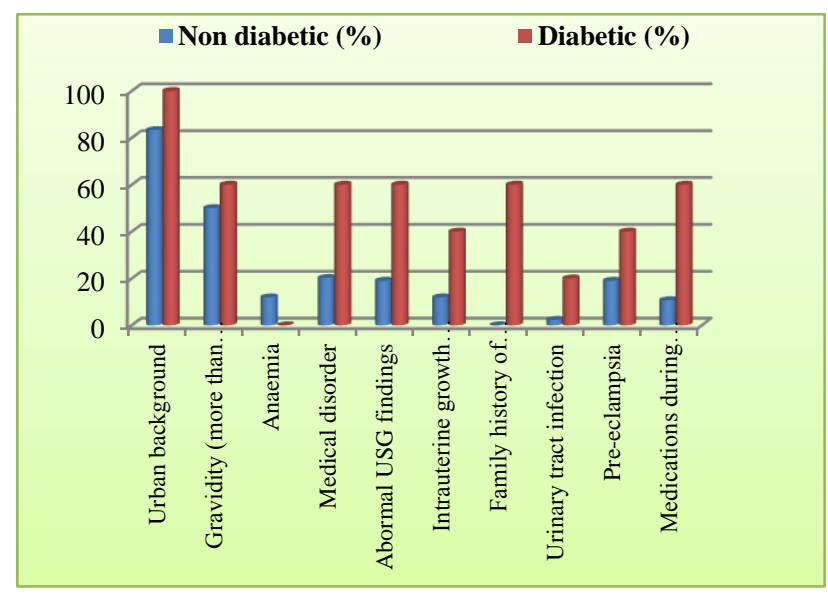

Figure 1: Comparison of participants with and without abnormal blood sugar in antenatal phase.

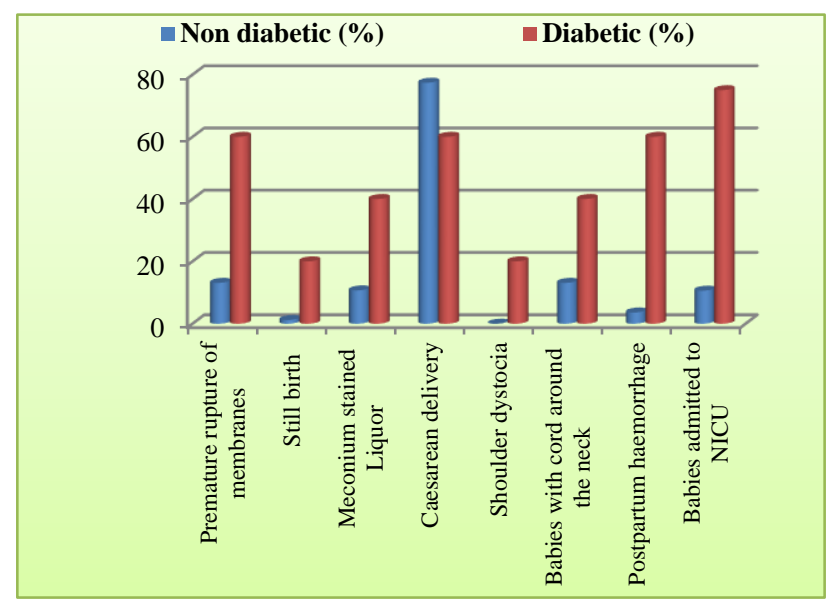

Figure 2: Comparison of participants with and without abnormal blood sugar in intranatal phase.
The findings of participants with and without abnormal blood sugar were compared antenatal phase (Figure 1) and intranatal phase (Figure 2). Although all the participants who had abnormal blood sugar levels required caesarean section, one could not be operated upon because she reported when foetal demise had already occurred, very late in labour when delivery was imminent. Another participant, who was kept was elective caesarean section delivered IUGR twins by precipitate labour before she could be taken for the section.

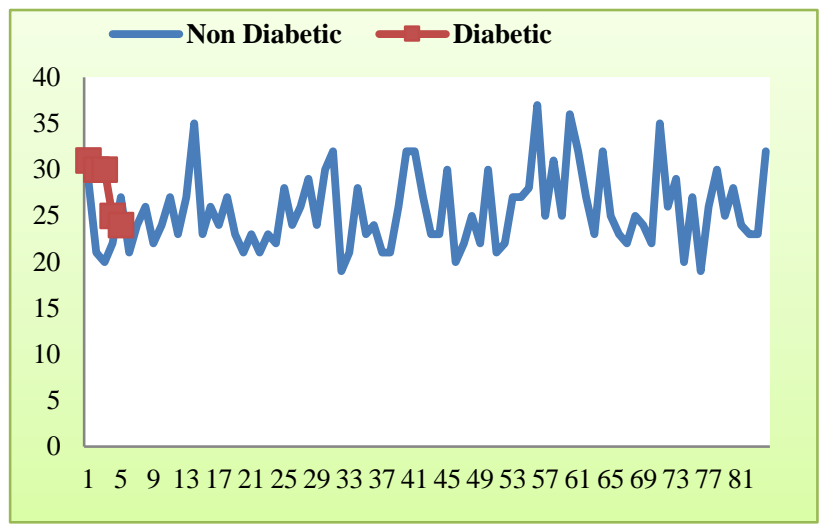

Figure 3: Comparison of age of participants with and without abnormal blood sugar.

The participants with diabetes were of relatively higher age range (Figure 3), delivered before 40 weeks of gestation (Figure 4) and had babies in higher range of birth weight (Figure 5). There were 3 participants with gestational DM.

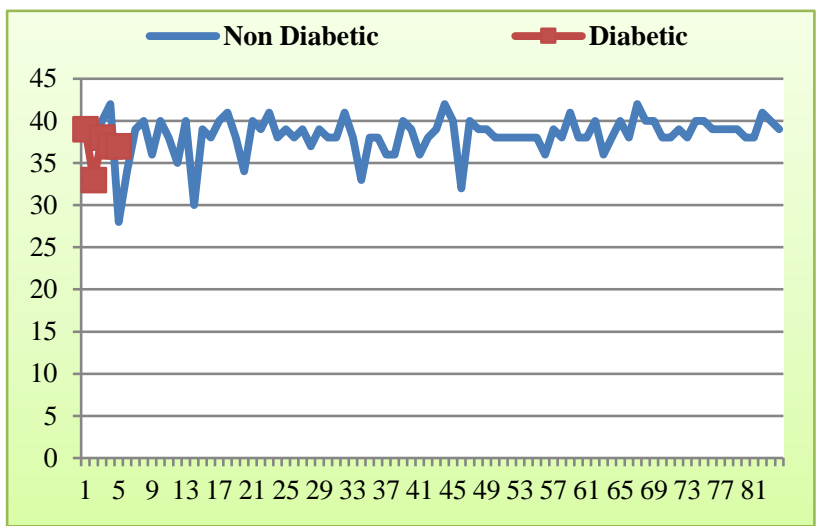

Figure 4: Comparison of gestational period of participants with and without abnormal blood sugar.

One participant with gestational DM who had good compliance could be managed with diet and had a good outcome while the other with poor compliance had poor outcome. She was physician by profession, reported at 6 weeks of conception when blood sugar was normal. Her father was having Type 2 diabetes; she was mildly obese and had history of polycystic ovarian disorder. Hence a high degree of suspicion for gestational diabetes was kept 
and regular foetal monitoring was done with ultrasound, anomaly scan, and triple test as per protocol. All the findings were normal. She was subjected to glucose tolerance test at 24 weeks that gave abnormal blood sugar values in mg/dL as 98 fasting, 193 after 1 hour, 163 after 2 hours, 138 after 3 hours.

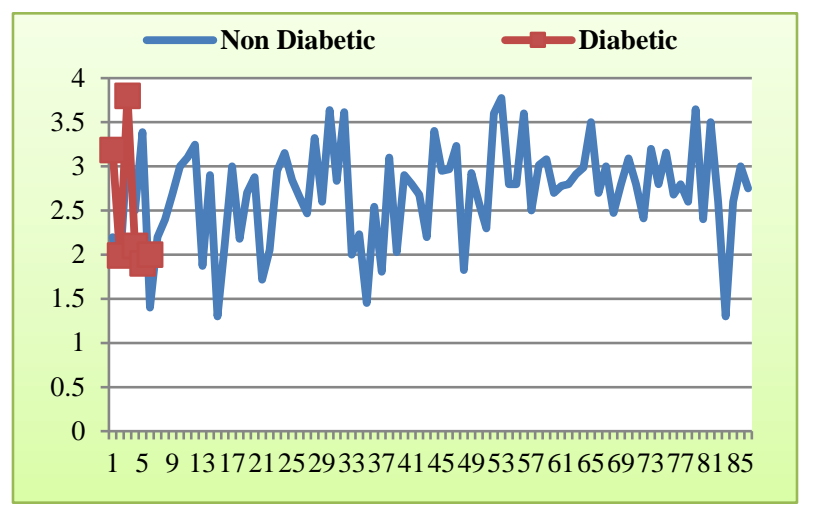

Figure 5: Comparison of baby weight of participants with and without abnormal blood sugar.

She was diagnosed as gestational DM and started with dietary control and exercise regime under consultation with medicine specialist. She followed all the advice and regularly reported for follow up. She had recurrent furunclosis during 32 weeks to 36 weeks of pregnancy that was treated with Amoxicillin Clavulanic Acid combination. Her blood sugar control was good and HbA1c was 6.2. Foetal growth and amount of liquor amnii on serial ultrasound examinations remained normal and thus she was reassured. The ultrasound examination showed cord around neck but foetal Doppler was normal. Per vaginal findings showed mild cephalo-pelvic disproportion and unripe cervix. Hence elective caesarean section was done at 39 weeks. She had mild atonic post partum haemorrhage that was controlled. The baby had birth weight of $3.185 \mathrm{Kg}$ and had an uneventful neonatal course. The second, who was a nurse by profession, had reported at 30 weeks with history of decreased foetal movements for one day. Her foetal cardiotocogram was found reactive and ultrasonography was found normal. She was diagnosed as having pregnancy induced hypertension and hence put on Alphadopa. Her random blood sugar was raised and hence glucose tolerance test was advised. She reported back with reports after gap of 6 weeks showing abnormal test results showing blood sugar values in mg/dL as 70 fasting, 196 after 1 hour, 159 after 2 hours, 100 after 3 hours. She was admitted immediately but absconded the next day.

Finally at gestational age of 38 weeks 2 days, she reported in emergency with history of no foetal movements for 3 days and leaking for 1 day. As the foetal heart sounds were not located and head was at vulva, she could not be taken for caesarean section. The labour was complicated by shoulder dystocia that was managed by McRobert's manoeuvre. The third participant with gestational DM was admitted as a precautionary measure at 32 weeks of gestation. She had history of rupture of one horn of bicornuate uterus in her previous pregnancy. She had normal blood sugar levels during early pregnancy but now the fasting and post meal blood sugar was abnormal and hence she was put on sliding scale of Insulin. She was positive for $\mathrm{HBsAg}$ and developed PPROM at 33 weeks and hence emergency caesarean was performed on emergency basis. She delivered a baby with birth weight of $1.99 \mathrm{Kg}$ who required admission to NICU. She had mild atonic PPH that was managed. Rest of the postnatal period was uneventful.

Two of the participants had pregestational diabetes and were on insulin supplements. One of them had history of third trimester intrauterine death in previous pregnancy and had twin pregnancy during this study. She was kept on foetal monitoring by serial ultrasonography as routine and admitted at 30 weeks of pregnancy. She had less than satisfactory blood sugar control with $\mathrm{HbAlc}$ value of $8.4 \mathrm{mg} / \mathrm{dL}$. She had urinary tract infection in the last trimester that was managed with antibiotics. Elective caesarean section was planned at 37 weeks but she developed PROM and went into preterm labour on previous night. She delivered twins with 2.1 and $1.9 \mathrm{Kg}$ birth weight vaginally before she could be shifted to operation theatre. The first of the twin had thick meconium stained liquor and friable umbilical cord suggesting chorioamnionitis.

The second had clear liquor and normal cord. Both babies were shifted to NICU. Another participant with pregestational DM had an unplanned pregnancy and conceived with short interconceptional period of 4 months. She was on high dose of Insulin and had good glycaemic control with HbA1c of $6.37 \mathrm{mg} / \mathrm{dL}$. Her insulin requirement increased in second trimester. She had preexisting medical conditions of hypothyroidism hypertension and proteinuria which increased in the second trimester. She was on thyroxine supplements and Amlodepine. She was kept on foetal monitoring with serial ultrasonography and Doppler study. Based on the findings, decision for elective caesarean section was taken at 37 weeks of gestation. She delivered an IUGR baby with weight $2 \mathrm{~kg}$ who required NICU admission.

\section{DISCUSSION}

This hospital based observational study of DM during pregnancy was done over a duration of $1 \frac{1 / 2}{2}$ year that included both gestational and pre-gestational DM. After comparing the statistical findings with previous studies, we have presented minute details of these participants that raise various issues in effective management of DM during pregnancy.

Swami et al found $7.7 \%$ incidence of gestational DM in Western India hospital based study. ${ }^{7}$ Raja et al found the prevalence rate of gestational DM was $7.8 \%$ and all the cases were newly diagnosed during the study. Among various risk factors associated significantly with GDM 
were gravid status, Parity and History of abortion. ${ }^{8}$ In present study, $3.3 \%$ incidence of gestational DM was found. Jadhao, Gawade and Ughade in an observational descriptive study of 214 high risk pregnancies in rural part of Central India found that gestational diabetes was present in 2 cases $(0.93 \%) .{ }^{9}$ Present study resulted with $5.7 \%$ prevalence of DM during pregnancy.

It is strongly advised that the preparation of women with type 1 diabetes for pregnancy should begin at least 6 months before conception. There are several measures that should be undertaken during this period to achieve recommended glycaemic control. ${ }^{10}$ However, both our diabetic participants reported with unplanned pregnancy.

Weissgerber and Mudd found that pre-existing diabetes is a risk factor for preeclampsia and worsening proteinuria during pregnancy is common in women with diabetic nephropathy due to increases in the glomerular filtration rate. They also found that obesity is a shared risk factor for both preeclampsia and type 2 diabetes; however, there is greater preeclampsia risk among women with type 2 diabetes. ${ }^{11} \mathrm{We}$ found that incidence of pre-eclampsia was $19 \%$ among non diabetic and $40 \%$ among diabetic participants. Two of the non-diabetic participants, who were not booked in antenatal care, presented with eclampsia and HELLP syndrome respectively. In one of our participants, proteinuria worsened in pregnancy with pregestational diabetes.

Gizzo et al found higher frequency of preterm delivery in pregestational diabetic women and inferred that pregestational diabetes could be directly responsible for ruptures of membranes and preterm delivery by amniotic fluid alteration and increased risk of ascending infections. They also detected a mean prevalence of macrosomia in $21-22 \%$ pregestational diabetics. ${ }^{12}$ In present study, $60 \%$ participants had premature rupture of membranes in diabetic population as against $13 \%$ in non-diabetic and $20 \%$ had macrosomia.

Buchanan et al suggested that management of diabetes in pregnancy shall include reducing caloric intake for overweight and obese women, limiting carbohydrate content to $35-40 \%$ of total calories and focusing on complex rather than simple carbohydrates. The second of these modifications has been shown to improve perinatal outcomes compared with higher carbohydrate diets. ${ }^{13}$ In present study we advised the same to diabetic pregnant mothers. One of the diabetic participants complied strictly as per dietary advice and exercise and could be managed well without insulin.

Gutaj and Wender have advised that prevention of intrauterine grown retardation (IUGR) should ideally start before pregnancy in patients of pregestational diabetes. Strict glycaemic control and intensive treatment of nephropathy and hypertension are essential. ${ }^{14} \mathrm{We}$ had two participants with pregestational diabetes both having unplanned pregnancy. However we ensured strict glycaemic control and managed hypertension to optimize obstetric outcomes.

Kamana et al have stated that the risk of postpartum bleeding and genital tract injury was about 3-5 times higher in macrosomic deliveries. ${ }^{15}$ In present study one patient with gestational diabetes had macrosomic baby and she had genital wall lacerations due to vaginal delivery.

Hall et al have studies pregestational and gestational DM in different groups and found that out of the 278 cases analysed, $60 \%$ had gestational, $40 \%$ pregestational DM. They found $6.5 \%$ intrauterine deaths in pregestational Type 2 DM but $12 \%$ intrauterine deaths in gestational DM. They found chronic hypertension in $23 \%$ of gestational DM and $42 \%$ of type 2 DM group. In gestational DM group, lifestyle modification and metformin sufficed in $88 \%$ cases and $12 \%$ required Insulin. However, Insulin was required in $53 \%$ of pregnancies with type $2 \mathrm{DM}$ group. They found that in case with intrauterine death, the mother defaulted on attending appointments and had given a history of reduced foetal movement. They found incidence of shoulder dystocia in $2.4 \%$ of gestational DM. $1 \%$ patients of Type 1 and Type $2 \mathrm{DM}$ each reported to them for preconception counselling. ${ }^{16}$

Present study also gave the same results with $60 \%$ gestational diabetes and $40 \%$ pregestational diabetes. There was only one still birth in present study that may be attributed to non-compliance of the participant to ideal management of DM during pregnancy. Present study had $2(40 \%)$ participants with hypertension where one had pre-existing hypertension. In present study, lifestyle modification sufficed in $1(20 \%)$, Insulin was required in second $(20 \%)$ while the third $(20 \%)$ was non-compliant for any kind of treatment. Among the pregestational diabetes, both $(100 \%)$ were already on Insulin and its requirement increased in pregnancy. We found shoulder dystocia in 1 participant $(20 \%)$ although it could have been prevented with caesarean in case the participant had reported in time. None of pregestational participants in present study had reported for preconception counselling.

There is lack of awareness regarding serious outcomes of diabetes in pregnancy and widespread ignorance regarding its management through diet, exercise and insulin supplementation. Health education for diabetes shall include special impetus on gestational diabetes as well as preconception planning and management of diabetes in pregnancy. We recommend further qualitative studies to assess and improve on patient compliance for treatment of diabetes.

\section{ACKNOWLEDGMENTS}

Authors acknowledge the participants for their contribution for this study. Authors also acknowledge Dr. Ajay Goenka, Chairman, Chirayu Medical College and 
Hospital, Bhopal for his support. Authors would like to thank Dr. Shashikala Jawade, Professor and Ex-Head, and Dr. Manik Sirpurkar, Professor, Department of Obstetrics and Gynaecology, Chirayu Medical College and Hospital, Bhopal for their guidance during this study.

\section{Funding: No funding sources}

Conflict of interest: None declared

Ethical approval: The study was approved in accordance with WMA declaration of Helsinki

\section{REFERENCES}

1. World Health Organization.Diabetes country profiles, 2016 - India. Available at http://www.who.int/diabetes/country-

profiles/ind_en.pdf?ua=1. Accessed on $2^{\text {nd }}$ July 2017.

2. ICMR Guidelines for management of Type 2 Diabetes - 2005. Available at http://icmr.nic.in/guidelines_diabetes/section1.pdf. Accessed on $2^{\text {nd }}$ July 2017.

3. Lumsden MA, A Ang C, Howe D, Lumsden M. Diabetes. In: James DK, Steer PJ, Weiner CP, Goni B editors. High risk pregnancy management options. $3^{\text {rd }}$ Ed. Elsevier; 986-1004.

4. Daftary SN, Bhide AG. Diabetes and pregnancy. In: Fernando Arias. Practical guide to high-risk pregnancy and delivery: A south Asian perspective. 3rd Ed. Elsevier; 440-64.

5. Berger H, Crane J, Farine D, Armson A, De La Ronde S, Keenan-Lindsay L et al. Screening for gestational diabetes mellitus. J Obstet Gynecol Can. 2002;24(11):894-912.

6. Carpenter MW, Coustan DR. Criteria for screening tests for gestational diabetes. Am J Obstet Gynecol 1982;144:768-73.

7. Swami SR, Mehetre R, Shivane V, Bandgar TR, Menon PS, Shah NS. Prevalence of carbohydrate intolerance of varying degrees in pregnant females in western India (Maharashtra): a hospital-based study. J Indian Med Assoc. 2008;106(11):712-4.
8. Raja MW, Baba TA, Hanga AJ, Bilquees S, Rasheed S, Ul Haq I et al. A study to estimate the prevalence of gestational diabetes mellitus in an urban block of Kashmir valley (North India). Int J Med Sci Public Health. 2014;3:191-5.

9. Jadhao AR, Gawade MD, Ughade SN. Outcome of pregnancy among high risk pregnancies in rural area of Nagpur, Maharashtra, Central India. Int J Community Med Public Health. 2017;4:628-33.

10. Cyganek K, Klupa T, Szopa M, Katra B, Małecki MT. Medical care of pregnant women with type 1 diabetes: current guidelines and clinical practice. Pol Arch Med Wewn. 2013;123(1-2):59-65.

11. Weissgerber TL, Mudd LM. Preeclampsia and Diabetes. Curr Diab Rep. 2015;15(3):579.

12. Gizzo S, Patrelli TS, Rossanese M, Noventa M, Berretta R, Di Gangi S et al. An update on diabetic women obstetrical outcomes linked to preconception and pregnancy glycemic profile: a systematic literature review. Scientific World J. 2013;2013.

13. Buchanan TA, Xiang AH, Page KA. Gestational diabetes mellitus: risks and management during and after pregnancy. Nat Rev Endocrinol. 2012;8(11):639-649.

14. Gutaj P, Wender-Ozegowska E. Diagnosis and management of IUGR in pregnancy complicated by type 1 diabetes mellitus. Curr Diab Rep. 2016;16:39.

15. Kamana KC, Shakya S, Zhang H. Gestational Diabetes Mellitus and Macrosomia: A Literature Review Ann Nutr Metab 2015;66(suppl 2):14-20.

16. Hall D, Du Toit M, Mason D, Conradie M. Diabetes mellitus in pregnancy, still changing. J Endocrinol, Metab Diab South Africa. 2015;20(3):108-14.

Cite this article as: Joshi PG, Joshi GA. Prevalence, clinical presentation and complications of diabetes mellitus in obstetric patients attending a medical college hospital. Int J Reprod Contracept ObstetGynecol 2017;6:4028-34. 